\title{
The convergence of the modified Mann and Ishikawa iterations in Banach spaces
}

Zhiqun Xue* and Guiwen LV

"Correspondence: xuezhiqun@126.com

Department of Mathematics and Physics, Shijiazhuang Tiedao

University, Shijiazhuang, 050043, P.R. China

\begin{abstract}
In this paper, under the new condition we show that the convergence of the modified Mann and Ishikawa iterations is equivalent for uniformly L-Lipschitz asymptotically pseudocontractive mappings in real Banach spaces. Our results extend and improve the corresponding results of Zeng (Acta Math. Sin. 47:219-228, 2004).

MSC: $47 \mathrm{H} 09 ; 47 \mathrm{H} 10$

Keywords: uniformly L-Lipschitz; asymptotically pseudocontractive mapping; fixed point; real Banach space
\end{abstract}

\section{Introduction and preliminaries}

Throughout the paper, we assume that $E$ is an arbitrary real Banach space, $D$ is a nonempty closed convex subset of $E, T: D \rightarrow D$ is a self-mapping and $F(T)$ is the fixed point set of $T$, i.e., $F(T)=\{x \in D: T x=x\}$. Let $J$ denote the normalized duality mapping from $E$ to $2^{E^{*}}$ defined by

$$
J(x)=\left\{f \in E^{*}:\langle x, f\rangle=\|x\|^{2}=\|f\|^{2}\right\}, \quad \forall x \in E,
$$

where $E^{*}$ denotes the dual space of $E$ and $\langle\cdot, \cdot\rangle$ denotes the generalized duality pairing. The single-valued normalized duality mapping is denoted by $j$.

Definition 1.1 (see [1]) (1) A mapping $T$ is said to be uniformly L-Lipschitz if there exists a constant $L>0$ such that, for all $x, y \in D$,

$$
\left\|T^{n} x-T^{n} y\right\| \leq L\|x-y\|, \quad \forall n \geq 1 .
$$

(2) The mapping $T$ is said to be asymptotically nonexpansive with a sequence $\left\{k_{n}\right\} \subset$ $[1,+\infty)$ and $\lim _{n \rightarrow \infty} k_{n}=1$ if, for all $x, y \in D$,

$$
\left\|T^{n} x-T^{n} y\right\| \leq k_{n}\|x-y\|, \quad \forall n \geq 1 .
$$

(3) The mapping $T$ is said to be asymptotically pseudocontractive with a sequence $\left\{k_{n}\right\} \subset$ $[1,+\infty)$ and $\lim _{n \rightarrow \infty} k_{n}=1$ if, for all $x, y \in D$, there exists $j(x-y) \in J(x-y)$ such that

$$
\left\langle T^{n} x-T^{n} y, j(x-y)\right\rangle \leq k_{n}\|x-y\|^{2}, \quad \forall n \geq 1 .
$$

๑ 2013 Xue and Lv; licensee Springer. This is an Open Access article distributed under the terms of the Creative Commons Attribution License (http://creativecommons.org/licenses/by/2.0), which permits unrestricted use, distribution, and reproduction in any medium, provided the original work is properly cited. 
Obviously, an asymptotically nonexpansive mapping is both asymptotically pseudocontractive and uniformly $L$-Lipschitz, but the converse is not true in general. For more details on uniformly $L$-Lipschitz asymptotically nonexpansive and asymptotically pseudocontractive mappings, see [2-6] and [7-11].

Definition 1.2 (see [1]) For any $u_{1}, x_{1} \in D$, the sequences $\left\{u_{n}\right\}$ and $\left\{x_{n}\right\}$ in $D$ defined by

$$
u_{n+1}=\left(1-a_{n}\right) u_{n}+a_{n} T^{n} u_{n}, \quad \forall n \geq 1,
$$

and

$$
\left\{\begin{array}{l}
y_{n}=\left(1-b_{n}\right) x_{n}+b_{n} T^{n} x_{n}, \\
x_{n+1}=\left(1-a_{n}\right) x_{n}+a_{n} T^{n} y_{n}, \quad \forall n \geq 1,
\end{array}\right.
$$

are called the modified Mann and Ishikawa iterations, respectively, where $\left\{a_{n}\right\},\left\{b_{n}\right\}$ are two real sequences in $[0,1]$ satisfying some conditions. For more details on the Mann and Ishikawa iterations, see $[4,12]$ and $[11]$.

In 2001, Chidume and Mutangadura [13] constructed an example for every nontrivial Mann iteration failing to converge while Ishikawa iteration converges. Therefore, there exist some differences between convergence of two kinds of the iterative sequences. Since then, many authors have shown that the Mann (modified Mann) and Ishikawa (modified Ishikawa) iterations (with errors) converge strongly to fixed points of pseudocontractive mappings and others under appropriate conditions.

Especially, Chang [1] proved the following.

Theorem 1.3 [1, Theorem 2.1] Let $D$ be a nonempty closed convex subset of $E$ and $T$ : $D \rightarrow D$ be a uniformly L-Lipschitzian asymptotically pseudocontractive mapping with a sequence $\left\{k_{n}\right\} \subset[1,+\infty)$ such that $\lim _{n \rightarrow \infty} k_{n}=1$ and $L \geq 1$. Let $\left\{a_{n}\right\}$ and $\left\{b_{n}\right\}$ be two real sequences in $[0,1]$ satisfying the following conditions:

(a) $a_{n}, b_{n} \rightarrow 0$ as $n \rightarrow \infty$;

(b) $\sum_{n=0}^{\infty} a_{n}=\infty$.

For any $x_{0} \in D$, let $\left\{x_{n}\right\}$ be the modified Ishikawa iteration defined by (1.2). If $F(T) \neq \emptyset$, $q \in F(T)$ and there exists a strictly increasing function $\Phi:[0,+\infty) \rightarrow[0,+\infty)$ with $\Phi(0)=0$ such that

$$
\left\langle T^{n} x_{n+1}-q, j\left(x_{n+1}-q\right)\right\rangle \leq k_{n}\left\|x_{n+1}-q\right\|^{2}-\Phi\left(\left\|x_{n+1}-q\right\|\right), \quad \forall n \geq 0,
$$

where $j\left(x_{n+1}-q\right) \in J\left(x_{n+1}-q\right)$, then $\left\{x_{n}\right\}$ converges strongly to a fixed point $q$ of $T$.

Theorem 1.4 [1, Theorem 2.3] Let $D$ be a nonempty closed convex subset of $E$ and $T$ : $D \rightarrow D$ be a uniformly L-Lipschitzian asymptotically pseudocontractive mapping with a sequence $\left\{k_{n}\right\} \subset[1,+\infty)$ such that $\lim _{n \rightarrow \infty} k_{n}=1$ and $L \geq 1$. Let $\left\{a_{n}\right\}$ be the real sequence in $[0,1]$ satisfying the following conditions:

(a) $a_{n} \rightarrow 0$ as $n \rightarrow \infty$;

(b) $\sum_{n=0}^{\infty} a_{n}=\infty$. 
For any $u_{0} \in D$, let $\left\{u_{n}\right\}$ be the modified Mann iteration defined by (1.1). If $F(T) \neq \emptyset$, $q \in F(T)$ and there exists a strictly increasingfunction $\Phi:[0,+\infty) \rightarrow[0,+\infty)$ with $\Phi(0)=0$ satisfying the condition (2.1) of [1, Theorem 2.1], then $\left\{u_{n}\right\}$ converges strongly to a fixed point $q$ of $T$.

Motivated by Theorems 1.3 and 1.4, Zeng [14] gave another interesting results as follows.

Theorem 1.5 [14, Theorem 2.1] Let $D$ be a nonempty closed convex subset of $E$ and $T: D \rightarrow D$ be a uniformly L-Lipschitz asymptotically pseudocontractive mapping with a sequence $\left\{k_{n}\right\} \subset[1,+\infty)$ such that $\lim _{n \rightarrow \infty} k_{n}=1$ and $L \geq 1$. Let $\left\{a_{n}\right\}$ and $\left\{b_{n}\right\}$ be two real sequences in $[0,1]$ satisfying the following conditions:

(a) $a_{n} \rightarrow 0$ as $n \rightarrow \infty$ and $\sum_{n=0}^{\infty} a_{n}=\infty$;

(b) $\sum_{n=0}^{\infty} a_{n}^{2}<\infty$ and $\sum_{n=0}^{\infty} a_{n}\left(k_{n}-1\right)<\infty$;

(c) $\sum_{n=0}^{\infty} a_{n} b_{n}<\infty$.

For arbitrary $x_{0} \in D$, let $\left\{x_{n}\right\}$ be the modified Ishikawa iteration defined by (1.2). If $F(T) \neq \emptyset, q \in F(T)$ and there exists a strictly increasing function $\Phi:[0,+\infty) \rightarrow[0,+\infty)$ with $\Phi(0)=0$ such that

$$
\left\langle T^{n} x_{n+1}-q, j\left(x_{n+1}-q\right)\right\rangle \leq k_{n}\left\|x_{n+1}-q\right\|^{2}-\Phi\left(\left\|x_{n+1}-q\right\|\right), \quad \forall n \geq 0
$$

where $j\left(x_{n+1}-q\right) \in J\left(x_{n+1}-q\right)$, then $\left\{x_{n}\right\}$ converges strongly to the fixed point $q$ of $T$.

Theorem 1.6 [14, Theorem 2.3] Let D be a nonempty closed convex subset of $E$ and $T: D \rightarrow D$ be a uniformly L-Lipschitz asymptotically pseudocontractive mapping with a sequence $\left\{k_{n}\right\} \subset[1,+\infty)$ such that $\lim _{n \rightarrow \infty} k_{n}=1$ and $L \geq 1$. Let $\left\{a_{n}\right\}$ be a real sequence in $[0,1]$ satisfying the following conditions:

(a) $a_{n} \rightarrow 0$ as $n \rightarrow \infty$ and $\sum_{n=0}^{\infty} a_{n}=\infty$;

(b) $\sum_{n=0}^{\infty} a_{n}^{2}<\infty$ and $\sum_{n=0}^{\infty} a_{n}\left(k_{n}-1\right)<\infty$.

For arbitrary $u_{0} \in D$, let $\left\{u_{n}\right\}$ be the modified Mann iteration defined by (1.1). If $F(T) \neq \emptyset$, $q \in F(T)$ and there exists a strictly increasing function $\Phi:[0,+\infty) \rightarrow[0,+\infty)$ with $\Phi(0)=0$ satisfying the condition (2.1) of [1, Theorem 2.1], then $\left\{u_{n}\right\}$ converges strongly to the fixed point $q$ of $T$.

It is worth mentioning that the result of Chang [1] is different from that of Zeng [14]. This can be seen from the following example.

\section{Example 1.7 Set}

$$
a_{n}=\left\{\begin{array}{ll}
0, & n=2 i, \\
\frac{1}{n}, & n=2 i-1,
\end{array} \quad b_{n}=\left\{\begin{array}{ll}
\frac{1}{2}, & n=2 i, \\
\frac{1}{n}, & n=2 i-1,
\end{array} \quad k_{n}=1+\frac{1}{n}, \quad \forall i \geq 1, n \geq 1 .\right.\right.
$$

Then $a_{n} \rightarrow 0$ as $n \rightarrow \infty, \sum_{n=1}^{\infty} a_{n}=\infty$ and $\sum_{n=1}^{\infty} a_{n}^{2}<\infty, \sum_{n=1}^{\infty} a_{n} b_{n}<\infty, \sum_{n=1}^{\infty} a_{n}\left(k_{n}-1\right)<$ $\infty$, but $b_{n} \rightarrow 0$ as $n \rightarrow \infty$ does not hold. On the other hand, let

$$
a_{n}=\left\{\begin{array}{ll}
0, & n=2 i, \\
\frac{1}{\sqrt{n}}, & n=2 i-1,
\end{array} \quad b_{n}=\left\{\begin{array}{ll}
0, & n=2 i, \\
\frac{1}{\sqrt{n}}, & n=2 i-1,
\end{array} \quad k_{n}=1+\frac{1}{\sqrt{n}}, \quad \forall i \geq 1, n \geq 1 .\right.\right.
$$


Then $a_{n}, b_{n} \rightarrow 0$ as $n \rightarrow \infty$ and $\sum_{n=1}^{\infty} a_{n}=\infty$, but $\sum_{n=1}^{\infty} a_{n}^{2}=\infty, \sum_{n=1}^{\infty} a_{n} b_{n}=\infty$ and $\sum_{n=1}^{\infty} a_{n}\left(k_{n}-1\right)=\infty$.

The aim of this paper is to extend and improve Theorem 1.5 and Theorem 1.6.

For this, we need to use the following lemmas.

Lemma 1.8 [1] Let $E$ be a real Banach space and $J: E \rightarrow 2^{E^{*}}$ be a normalized duality mapping. Then, for all $x, y \in E$ and $j(x+y) \in J(x+y)$,

$$
\|x+y\|^{2} \leq\|x\|^{2}+2\langle y, j(x+y)\rangle
$$

Lemma 1.9 [14] Let $\left\{a_{n}\right\},\left\{b_{n}\right\}$ and $\left\{c_{n}\right\}$ be three nonnegative real sequences satisfying

$$
a_{n+1} \leq\left(1+b_{n}\right) a_{n}+c_{n}, \quad \forall n \geq 0 .
$$

If $\sum_{n=0}^{\infty} b_{n}<\infty, \sum_{n=0}^{\infty} c_{n}<\infty$, then $\lim _{n \rightarrow \infty} a_{n}$ exists.

\section{Main results}

Now, we give the main results in this paper.

Theorem 2.1 Let $D$ be a nonempty closed convex subset of $E$ and $T: D \rightarrow D$ be a uniformly L-Lipschitz asymptotically pseudocontractive mapping with a sequence $\left\{k_{n}\right\} \subset[1,+\infty)$ such that $\lim _{n \rightarrow \infty} k_{n}=1$ and $L \geq 1$. Let $\left\{a_{n}\right\}$ be a real sequence in $[0,1]$ satisfying the following conditions:

(a) $a_{n} \rightarrow 0$ as $n \rightarrow \infty$ and $\sum_{n=1}^{\infty} a_{n}=\infty$;

(b) $\sum_{n=1}^{\infty} a_{n}^{2}<\infty$ and $\sum_{n=1}^{\infty} a_{n}\left(k_{n}-1\right)<\infty$.

For arbitrary $u_{1} \in D$, let $\left\{u_{n}\right\}$ be the modified Mann iteration defined by (1.5). If $F(T) \neq \emptyset$, $q \in F(T)$ and there exists a strictly increasing continuous function $\Phi:[0,+\infty) \rightarrow[0,+\infty)$ with $\Phi(0)=0$ such that

$$
\left\langle T^{n} u_{n+1}-q, j\left(u_{n+1}-q\right)\right\rangle \leq k_{n}\left\|u_{n+1}-q\right\|^{2}-\frac{\Phi\left(\left\|u_{n+1}-q\right\|\right)}{1+\Phi\left(\left\|u_{n+1}-q\right\|\right)+\left\|u_{n+1}-q\right\|^{2}}, \quad \forall n \geq 1,
$$

where $j\left(u_{n+1}-q\right) \in J\left(u_{n+1}-q\right)$, then $\left\{u_{n}\right\}$ converges strongly to a fixed point $q$ of $T$.

Proof Applying (1.5) and Lemma 1.8, we have

$$
\begin{aligned}
\left\|u_{n+1}-q\right\|^{2}= & \left\|\left(1-a_{n}\right)\left(u_{n}-q\right)+a_{n}\left(T^{n} u_{n}-q\right)\right\|^{2} \\
\leq & \left(1-a_{n}\right)^{2}\left\|u_{n}-q\right\|^{2}+2 a_{n}\left\langle T^{n} u_{n}-q, j\left(u_{n+1}-q\right)\right\rangle \\
= & \left(1-a_{n}\right)^{2}\left\|u_{n}-q\right\|^{2}+2 a_{n}\left\langle T^{n} u_{n}-T^{n} u_{n+1}, j\left(u_{n+1}-q\right)\right\rangle \\
& +2 a_{n}\left\langle T^{n} u_{n+1}-T^{n} q, j\left(u_{n+1}-q\right)\right\rangle \\
\leq & \left(1-a_{n}\right)^{2}\left\|u_{n}-q\right\|^{2}+2 a_{n} L\left\|u_{n}-u_{n+1}\right\| \cdot\left\|u_{n+1}-q\right\| \\
& +2 a_{n}\left[k_{n}\left\|u_{n+1}-q\right\|^{2}-\frac{\Phi\left(\left\|u_{n+1}-q\right\|\right)}{1+\Phi\left(\left\|u_{n+1}-q\right\|\right)+\left\|u_{n+1}-q\right\|^{2}}\right] .
\end{aligned}
$$


Observe that

$$
\begin{aligned}
\left\|u_{n+1}-u_{n}\right\| & =\left\|a_{n}\left(T^{n} u_{n}-u_{n}\right)\right\| \\
& \leq a_{n}\left\|T^{n} u_{n}-T^{n} q+q-u_{n}\right\| \\
& \leq a_{n}(1+L)\left\|u_{n}-q\right\| .
\end{aligned}
$$

Substituting (2.2) into (2.1), we obtain

$$
\begin{aligned}
\left\|u_{n+1}-q\right\|^{2} \leq & \left(1-a_{n}\right)^{2}\left\|u_{n}-q\right\|^{2}+a_{n}^{2} L(1+L)\left(\left\|u_{n}-q\right\|^{2}+\left\|u_{n+1}-q\right\|^{2}\right) \\
& +2 a_{n}\left[k_{n}\left\|u_{n+1}-q\right\|^{2}-\frac{\Phi\left(\left\|u_{n+1}-q\right\|\right)}{1+\Phi\left(\left\|u_{n+1}-q\right\|\right)+\left\|u_{n+1}-q\right\|^{2}}\right] .
\end{aligned}
$$

Since $a_{n} \rightarrow 0$ and $k_{n} \rightarrow 1$ as $n \rightarrow \infty$, without loss of generality, we assume that

$$
\frac{1}{2}<1-a^{2} L(1+L)-2 a_{n} k_{n}<1, \quad \forall n \geq 1
$$

Then (2.3) implies that

$$
\begin{aligned}
\left\|u_{n+1}-q\right\|^{2} & \\
\leq & \frac{\left(1-a_{n}\right)^{2}+a_{n}^{2} L(1+L)}{1-a^{2} L(1+L)-2 a_{n} k_{n}}\left\|u_{n}-q\right\|^{2} \\
& -\frac{2 a_{n}}{1-a^{2} L(1+L)-2 a_{n} k_{n}} \cdot \frac{\Phi\left(\left\|u_{n+1}-q\right\|\right)}{1+\Phi\left(\left\|u_{n+1}-q\right\|\right)+\left\|u_{n+1}-q\right\|^{2}} \\
\leq & \left\{1+\frac{2 a_{n}\left(k_{n}-1\right)+a_{n}^{2}[1+2 L(1+L)]}{1-a^{2} L(1+L)-2 a_{n} k_{n}}\right\}\left\|u_{n}-q\right\|^{2}-\frac{2 a_{n} \Phi\left(\left\|u_{n+1}-q\right\|\right)}{1+\Phi\left(\left\|u_{n+1}-q\right\|\right)+\left\|u_{n+1}-q\right\|^{2}} \\
\leq & \left\{1+4 a_{n}\left(k_{n}-1\right)+2 a_{n}^{2}[1+2 L(1+L)]\right\}\left\|u_{n}-q\right\|^{2}-\frac{2 a_{n} \Phi\left(\left\|u_{n+1}-q\right\|\right)}{1+\Phi\left(\left\|u_{n+1}-q\right\|\right)+\left\|u_{n+1}-q\right\|^{2}} \\
\leq & \left\{1+4 a_{n}\left(k_{n}-1\right)+2 a_{n}^{2}[1+2 L(1+L)]\right\}\left\|u_{n}-q\right\|^{2} .
\end{aligned}
$$

Since $\sum_{n=1}^{\infty}\left\{4 a_{n}\left(k_{n}-1\right)+2 a_{n}^{2}[1+2 L(1+L)]\right\}<\infty$, by Lemma $1.9, \lim _{n \rightarrow \infty}\left\|u_{n}-q\right\|$ exists. Denote $M=\sup _{n \geq 1}\left\{\left\|u_{n}-q\right\|\right\}$.

On the other hand, from (2.4), we have

$$
\begin{aligned}
& \left\|u_{n+1}-q\right\|^{2} \\
& \leq\left\{1+4 a_{n}\left(k_{n}-1\right)+2 a_{n}^{2}[1+2 L(1+L)]\right\}\left\|u_{n}-q\right\|^{2} \\
& \quad-\frac{2 a_{n} \Phi\left(\left\|u_{n+1}-q\right\|\right)}{1+\Phi\left(\left\|u_{n+1}-q\right\|\right)+\left\|u_{n+1}-q\right\|^{2}} \\
& \leq\left\|u_{n}-q\right\|^{2}+\left\{4 a_{n}\left(k_{n}-1\right)+2 a_{n}^{2}[1+2 L(1+L)]\right\} M^{2} \\
& \quad-\frac{2 a_{n} \Phi\left(\left\|u_{n+1}-q\right\|\right)}{1+\Phi\left(\left\|u_{n+1}-q\right\|\right)+\left\|u_{n+1}-q\right\|^{2}} .
\end{aligned}
$$

Let $\inf _{n \geq 1} \frac{\Phi\left(\left\|u_{n+1}-q\right\|\right)}{1+\Phi\left(\left\|u_{n+1}-q\right\|\right)+\left\|u_{n+1}-q\right\|^{2}}=\delta$. Then $\delta=0$. Assume $\delta>0$. Then we have

$$
\frac{\Phi\left(\left\|u_{n+1}-q\right\|\right)}{1+\Phi\left(\left\|u_{n+1}-q\right\|\right)+\left\|u_{n+1}-q\right\|^{2}} \geq \delta, \quad \forall n \geq 1
$$


It follows from (2.5) that

$$
2 a_{n} \delta \leq\left\|u_{n}-q\right\|^{2}-\left\|u_{n+1}-q\right\|^{2}+\left[4 a_{n}\left(k_{n}-1\right)+2 a_{n}^{2}(1+2 L(1+L))\right] M^{2},
$$

which implies that

$$
2 \delta \sum_{n=1}^{\infty} a_{n} \leq\left\|u_{1}-q\right\|^{2}+\sum_{n=1}^{\infty}\left[4 a_{n}\left(k_{n}-1\right)+2 a_{n}^{2}(1+2 L(1+L))\right] M^{2}<\infty
$$

which is a contradiction, and so $\delta=0$. Thus, there exists a subsequence

$$
\left\{\frac{\Phi\left(\left\|u_{n_{i}+1}-q\right\|\right)}{1+\Phi\left(\left\|u_{n_{i}+1}-q\right\|\right)+\left\|u_{n_{i}+1}-q\right\|^{2}}\right\}
$$

of

$$
\left\{\frac{\Phi\left(\left\|u_{n+1}-q\right\|\right)}{1+\Phi\left(\left\|u_{n+1}-q\right\|\right)+\left\|u_{n+1}-q\right\|^{2}}\right\}
$$

such that

$$
\lim _{i \rightarrow \infty} \frac{\Phi\left(\left\|u_{n_{i}+1}-q\right\|\right)}{1+\Phi\left(\left\|u_{n_{i}+1}-q\right\|\right)+\left\|u_{n_{i}+1}-q\right\|^{2}}=0 .
$$

Since $0 \leq\left\|u_{n}-q\right\| \leq M$, it follows that

$$
0 \leq \frac{\Phi\left(\left\|u_{n_{i}+1}-q\right\|\right)}{1+\Phi(M)+M^{2}} \leq \frac{\Phi\left(\left\|u_{n_{i}+1}-q\right\|\right)}{1+\Phi\left(\left\|u_{n_{i}+1}-q\right\|\right)+\left\|u_{n_{i}+1}-q\right\|^{2}}
$$

Thus, $\lim _{i \rightarrow \infty} \Phi\left(\left\|u_{n_{i}+1}-q\right\|\right)=0$. By the strictly increasing continuous function $\Phi$, we obtain that $\lim _{i \rightarrow \infty}\left\|u_{n_{i}+1}-q\right\|=0$ and so $\lim _{n \rightarrow \infty}\left\|u_{n}-q\right\|=0$. This completes the proof.

Theorem 2.2 Let E be a real Banach space and D be a nonempty closed convex subset of E. Let $T: D \rightarrow D$ be a uniformly L-Lipschitz asymptotically pseudocontractive mapping with a sequence $\left\{k_{n}\right\} \subset[1,+\infty)$ such that $\lim _{n \rightarrow \infty} k_{n}=1$. Suppose that $\left\{a_{n}\right\}$ and $\left\{b_{n}\right\}$ are two real sequences in $[0,1]$ satisfying the following conditions:

(a) $a_{n} \rightarrow 0$ as $n \rightarrow \infty$ and $\sum_{n=1}^{\infty} a_{n}=\infty$;

(b) $\sum_{n=1}^{\infty} a_{n}^{2}<\infty$ and $\sum_{n=1}^{\infty} a_{n}\left(k_{n}-1\right)<\infty$;

(c) $\sum_{n=1}^{\infty} a_{n} b_{n}<\infty$.

For any $u_{1}, x_{1} \in D$, let $\left\{u_{n}\right\}$ and $\left\{x_{n}\right\}$ be the modified Mann and Ishikawa iterations defined by (1.5) and (1.6), respectively. If $F(T) \neq \emptyset, q \in F(T)$ and there exists a strictly increasing continuous function $\Phi:[0,+\infty) \rightarrow[0,+\infty)$ with $\Phi(0)=0$ such that

$$
\begin{aligned}
& \left\langle T^{n} x_{n+1}-T^{n} u_{n+1}, j\left(x_{n+1}-u_{n+1}\right)\right\rangle \\
& \quad \leq k_{n}\left\|x_{n+1}-u_{n+1}\right\|^{2}-\frac{\Phi\left(\left\|x_{n+1}-u_{n+1}\right\|\right)}{1+\Phi\left(\left\|x_{n+1}-u_{n+1}\right\|\right)+\left\|x_{n+1}-u_{n+1}\right\|^{2}}
\end{aligned}
$$

where $j\left(x_{n+1}-u_{n+1}\right) \in J\left(x_{n+1}-u_{n+1}\right)$. Then the following two assertions are equivalent: 
(1) $\left\{u_{n}\right\}$ converges strongly to the fixed point $q$ of $T$.

(2) $\left\{x_{n}\right\}$ converges strongly to the fixed point $q$ of $T$.

Proof If the iteration (1.6) converges to a fixed point $q$, then, by putting $b_{n}=0$, we can get the convergence of the iteration (1.5).

Conversely, we only need to prove that the iteration (1.5) $\Rightarrow$ the iteration (1.6), i.e., $\| u_{n}-$ $q \| \rightarrow 0$ as $n \rightarrow \infty \Rightarrow\left\|x_{n}-q\right\| \rightarrow 0$ as $n \rightarrow \infty$. Here, without loss of generality, let $\| u_{n}-$ $q \| \leq 1$. Then $\left\|T^{n} u_{n}-u_{n}\right\| \leq(1+L)$.

Applying the iterations (1.5), (1.6) and Lemma 1.8, we have

$$
\begin{aligned}
\left\|x_{n+1}-u_{n+1}\right\|^{2}= & \left\|\left(1-a_{n}\right)\left(x_{n}-u_{n}\right)+a_{n}\left(T^{n} y_{n}-T^{n} u_{n}\right)\right\|^{2} \\
\leq & \left(1-a_{n}\right)^{2}\left\|x_{n}-u_{n}\right\|^{2}+2 a_{n}\left\langle T^{n} y_{n}-T^{n} u_{n}, j\left(x_{n+1}-u_{n+1}\right)\right\rangle \\
= & \left(1-a_{n}\right)^{2}\left\|x_{n}-u_{n}\right\|^{2}+2 a_{n}\left\langle T^{n} y_{n}-T^{n} x_{n+1}, j\left(x_{n+1}-u_{n+1}\right)\right\rangle \\
& +2 a_{n}\left\langle T^{n} x_{n+1}-T^{n} u_{n+1}, j\left(x_{n+1}-u_{n+1}\right)\right\rangle \\
& +2 a_{n}\left\langle T^{n} u_{n+1}-T^{n} u_{n}, j\left(x_{n+1}-u_{n+1}\right)\right\rangle \\
\leq & \left(1-a_{n}\right)^{2}\left\|x_{n}-u_{n}\right\|^{2}+2 a_{n} L\left\|y_{n}-x_{n+1}\right\| \cdot\left\|x_{n+1}-u_{n+1}\right\| \\
& +2 a_{n}\left[k_{n}\left\|x_{n+1}-u_{n+1}\right\|^{2}-\frac{\Phi\left(\left\|x_{n+1}-u_{n+1}\right\|\right)}{1+\Phi\left(\left\|x_{n+1}-u_{n+1}\right\|\right)+\left\|x_{n+1}-u_{n+1}\right\|^{2}}\right] \\
& +2 a_{n} L\left\|u_{n+1}-u_{n}\right\| \cdot\left\|x_{n+1}-u_{n+1}\right\| .
\end{aligned}
$$

Observe that

$$
\begin{aligned}
\left\|u_{n+1}-u_{n}\right\|= & \left\|a_{n}\left(T^{n} u_{n}-u_{n}\right)\right\| \\
\leq & a_{n}(1+L), \\
\left\|y_{n}-u_{n}\right\|= & \left\|\left(1-b_{n}\right)\left(x_{n}-u_{n}\right)+b_{n}\left(T^{n} x_{n}-u_{n}\right)\right\| \\
\leq & \left(1-b_{n}\right)\left\|x_{n}-u_{n}\right\|+b_{n}\left\|T^{n} x_{n}-T^{n} u_{n}\right\|+b_{n}\left\|T^{n} u_{n}-u_{n}\right\| \\
\leq & \left(1-b_{n}+b_{n} L\right)\left\|x_{n}-u_{n}\right\|+b_{n}(1+L) \\
\leq & \left(1+b_{n} L\right)\left\|x_{n}-u_{n}\right\|+b_{n}(1+L), \\
\left\|y_{n}-x_{n+1}\right\|= & \left\|b_{n}\left(T^{n} x_{n}-x_{n}\right)+a_{n}\left(x_{n}-T^{n} y_{n}\right)\right\| \\
\leq & b_{n}\left[\left\|T^{n} x_{n}-T^{n} u_{n}\right\|+\left\|T^{n} u_{n}-u_{n}\right\|+\left\|u_{n}-x_{n}\right\|\right] \\
& +a_{n}\left[\left\|x_{n}-u_{n}\right\|+\left\|u_{n}-T^{n} u_{n}\right\|+\left\|T^{n} u_{n}-T^{n} y_{n}\right\|\right] \\
\leq & b_{n}\left[(L+1)\left\|x_{n}-u_{n}\right\|+(L+1)\right] \\
& +a_{n}\left[\left\|x_{n}-u_{n}\right\|+(L+1)+L\left\|u_{n}-y_{n}\right\|\right] \\
\leq & b_{n}\left[(L+1)\left\|x_{n}-u_{n}\right\|+(L+1)\right] \\
& +a_{n}\left[\left\|x_{n}-u_{n}\right\|+(L+1)+L\left[\left(1+b_{n} L\right)\left\|x_{n}-u_{n}\right\|+b_{n}(1+L)\right]\right] \\
= & A_{n}\left\|x_{n}-u_{n}\right\|+B_{n},
\end{aligned}
$$


where $A_{n}=b_{n}(L+1)+a_{n}\left[1+L\left(1+b_{n} L\right)\right] \rightarrow 0$ and $B_{n}=b_{n}(L+1)+a_{n}(L+1)\left(1+L b_{n}\right) \rightarrow 0$ as $n \rightarrow \infty$. Substituting (2.9), (2.11) into (2.8), we obtain

$$
\begin{aligned}
&\left\|x_{n+1}-u_{n+1}\right\|^{2} \\
& \leq\left(1-a_{n}\right)^{2}\left\|x_{n}-u_{n}\right\|^{2}+2 a_{n} L\left(A_{n}\left\|x_{n}-u_{n}\right\|+B_{n}\right)\left\|x_{n+1}-u_{n+1}\right\|+2 a_{n}^{2} L(1+L) \\
& \cdot\left\|x_{n+1}-u_{n+1}\right\|+2 a_{n}\left[k_{n}\left\|x_{n+1}-u_{n+1}\right\|^{2}-\frac{\Phi\left(\left\|x_{n+1}-u_{n+1}\right\|\right)}{1+\Phi\left(\left\|x_{n+1}-u_{n+1}\right\|\right)+\left\|x_{n+1}-u_{n+1}\right\|^{2}}\right] \\
& \leq\left(1-a_{n}\right)^{2}\left\|x_{n}-u_{n}\right\|^{2}+a_{n} L A_{n}\left\|x_{n}-u_{n}\right\|^{2}+a_{n} L A_{n}\left\|x_{n+1}-u_{n+1}\right\|^{2} \\
&+a_{n} L B_{n}+a_{n} L B_{n}\left\|x_{n+1}-u_{n+1}\right\|^{2}+a_{n}^{2} L(1+L)\left\|x_{n+1}-u_{n+1}\right\|^{2}+a_{n}^{2} L(1+L) \\
&+2 a_{n}\left[k_{n}\left\|x_{n+1}-u_{n+1}\right\|^{2}-\frac{\Phi\left(\left\|x_{n+1}-u_{n+1}\right\|\right)}{1+\Phi\left(\left\|x_{n+1}-u_{n+1}\right\|\right)+\left\|x_{n+1}-u_{n+1}\right\|^{2}}\right] \\
&= {\left[\left(1-a_{n}\right)^{2}+a_{n} L A_{n}\right]\left\|x_{n}-u_{n}\right\|^{2}+\left[a_{n} L A_{n}+a_{n} L B_{n}+a_{n}^{2} L(1+L)\right.} \\
&\left.+2 a_{n} k_{n}\right]\left\|x_{n+1}-u_{n+1}\right\|^{2}+a_{n} L B_{n}+a_{n}^{2} L(1+L) \\
&-2 a_{n} \frac{\Phi\left(\left\|x_{n+1}-u_{n+1}\right\|\right)}{1+\Phi\left(\left\|x_{n+1}-u_{n+1}\right\|\right)+\left\|x_{n+1}-u_{n+1}\right\|^{2}} .
\end{aligned}
$$

Since $a_{n}, b_{n}, A_{n}, B_{n}, k_{n}-1 \rightarrow 0$ as $n \rightarrow \infty$, without loss of generality, we assume that

$$
\frac{1}{2}<1-a_{n} L A_{n}-a_{n} L B_{n}-a_{n}^{2} L(1+L)-2 a_{n} k_{n}<1, \quad \forall n \geq 1 .
$$

Then (2.12) implies that

$$
\begin{aligned}
\| x_{n+1}- & u_{n+1} \|^{2} \\
\leq & \frac{\left(1-a_{n}\right)^{2}+a_{n} L A_{n}}{1-a_{n} L A_{n}-a_{n} L B_{n}-a_{n}^{2} L(1+L)-2 a_{n} k_{n}}\left\|x_{n}-u_{n}\right\|^{2} \\
& +\frac{a_{n} L B_{n}+a_{n}^{2} L(1+L)}{1-a_{n} L A_{n}-a_{n} L B_{n}-a_{n}^{2} L(1+L)-2 a_{n} k_{n}} \\
& -\frac{2 a_{n}}{1-a_{n} L A_{n}-a_{n} L B_{n}-a_{n}^{2} L(1+L)-2 a_{n} k_{n}} \\
& \cdot \frac{\Phi\left(\left\|x_{n+1}-u_{n+1}\right\|\right)}{1+\Phi\left(\left\|x_{n+1}-u_{n+1}\right\|\right)+\left\|x_{n+1}-u_{n+1}\right\|^{2}} \\
\leq & \left\|x_{n}-u_{n}\right\|^{2}+\frac{2 a_{n} L A_{n}+a_{n} L B_{n}+a_{n}^{2}\left(1+L+L^{2}\right)+2 a_{n}\left(k_{n}-1\right)}{1-a_{n} L A_{n}-a_{n} L B_{n}-a_{n}^{2} L(1+L)-2 a_{n} k_{n}}\left\|x_{n}-u_{n}\right\|^{2} \\
& +2 a_{n} L B_{n}+2 a_{n}^{2} L(1+L)-\frac{2 a_{n} \Phi\left(\left\|x_{n+1}-u_{n+1}\right\|\right)}{1+\Phi\left(\left\|x_{n+1}-u_{n+1}\right\|\right)+\left\|x_{n+1}-u_{n+1}\right\|^{2}} \\
\leq & {\left[1+4 a_{n} L A_{n}+2 a_{n} L B_{n}+2 a_{n}^{2}\left(1+L+L^{2}\right)+4 a_{n}\left(k_{n}-1\right)\right]\left\|x_{n}-u_{n}\right\|^{2} } \\
& +2 a_{n} L B_{n}+2 a_{n}^{2} L(1+L)-\frac{2 a_{n} \Phi\left(\left\|x_{n+1}-u_{n+1}\right\|\right)}{1+\Phi\left(\left\|x_{n+1}-u_{n+1}\right\|\right)+\left\|x_{n+1}-u_{n+1}\right\|^{2}} \\
\leq & {\left[1+4 a_{n} L A_{n}+2 a_{n} L B_{n}+2 a_{n}^{2}\left(1+L+L^{2}\right)+4 a_{n}\left(k_{n}-1\right)\right]\left\|x_{n}-u_{n}\right\|^{2} } \\
& +2 a_{n} L B_{n}+2 a_{n}^{2} L(1+L) .
\end{aligned}
$$


Since

$$
\sum_{n=1}^{\infty}\left[4 a_{n} L A_{n}+2 a_{n} L B_{n}+2 a_{n}^{2}\left(1+L+L^{2}\right)+4 a_{n}\left(k_{n}-1\right)\right]<\infty
$$

and $\sum_{n=1}^{\infty}\left[2 a_{n} L B_{n}+2 a_{n}^{2} L(1+L)\right]<\infty$, by Lemma $1.9, \lim _{n \rightarrow \infty}\left\|x_{n}-u_{n}\right\|$ exists. Denote $M_{0}=\sup _{n \geq 1}\left\{\left\|x_{n}-u_{n}\right\|\right\}$.

On the other hand, from (2.13), it follows that

$$
\begin{aligned}
\| x_{n+1}- & u_{n+1} \|^{2} \\
\leq & {\left[1+4 a_{n} L A_{n}+2 a_{n} L B_{n}+2 a_{n}^{2}\left(1+L+L^{2}\right)+4 a_{n}\left(k_{n}-1\right)\right]\left\|x_{n}-u_{n}\right\|^{2} } \\
& +2 a_{n} L B_{n}+2 a_{n}^{2} L(1+L)-\frac{2 a_{n} \Phi\left(\left\|x_{n+1}-u_{n+1}\right\|\right)}{1+\Phi\left(\left\|x_{n+1}-u_{n+1}\right\|\right)+\left\|x_{n+1}-u_{n+1}\right\|^{2}} \\
\leq & \left\|x_{n}-u_{n}\right\|^{2}+\left[4 a_{n} L A_{n}+2 a_{n} L B_{n}+2 a_{n}^{2}\left(1+L+L^{2}\right)+4 a_{n}\left(k_{n}-1\right)\right] M^{2} \\
& +2 a_{n} L B_{n}+2 a_{n}^{2} L(1+L)-\frac{2 a_{n} \Phi\left(\left\|x_{n+1}-u_{n+1}\right\|\right)}{1+\Phi\left(\left\|x_{n+1}-u_{n+1}\right\|\right)+\left\|x_{n+1}-u_{n+1}\right\|^{2}} .
\end{aligned}
$$

Let $\inf _{n \geq 1} \frac{\Phi\left(\left\|x_{n+1}-u_{n+1}\right\|\right)}{1+\Phi\left(\left\|x_{n+1}-u_{n+1}\right\|\right)+\left\|x_{n+1}-u_{n+1}\right\|^{2}}=\delta$. Then $\delta=0$. Assume $\delta>0$. Then we have

$$
\frac{\Phi\left(\left\|x_{n+1}-u_{n+1}\right\|\right)}{1+\Phi\left(\left\|x_{n+1}-u_{n+1}\right\|\right)+\left\|x_{n+1}-u_{n+1}\right\|^{2}} \geq \delta, \quad \forall n \geq 1
$$

It follows from (2.14) that

$$
\begin{aligned}
2 a_{n} \delta \leq & \left\|x_{n}-u_{n}\right\|^{2}-\left\|x_{n+1}-u_{n+1}\right\|^{2} \\
& +\left[4 a_{n} L A_{n}+2 a_{n} L B_{n}+2 a_{n}^{2}\left(1+L+L^{2}\right)+4 a_{n}\left(k_{n}-1\right)\right] M^{2} \\
& +2 a_{n} L B_{n}+2 a_{n}^{2} L(1+L),
\end{aligned}
$$

which implies that

$$
\begin{aligned}
2 \delta \sum_{n=1}^{\infty} a_{n} \leq & \left\|x_{1}-u_{1}\right\|^{2}+\sum_{n=1}^{\infty}\left[4 a_{n} L A_{n}+2 a_{n} L B_{n}+2 a_{n}^{2}(1+L)+4 a_{n}\left(k_{n}-1\right)\right] M^{2} \\
& +\sum_{n=1}^{\infty}\left[2 a_{n} L B_{n}+2 a_{n}^{2} L(1+L)\right]<\infty
\end{aligned}
$$

which is a contradiction, and so $\delta=0$. Thus, there exists a subsequence

$$
\left\{\frac{\Phi\left(\left\|x_{n_{i}+1}-u_{n_{i}+1}\right\|\right)}{1+\Phi\left(\left\|x_{n_{i}+1}-u_{n_{i}+1}\right\|\right)+\left\|x_{n_{i}+1}-u_{n_{i}+1}\right\|^{2}}\right\}
$$

of

$$
\left\{\frac{\Phi\left(\left\|x_{n+1}-u_{n_{i}+1}\right\|\right)}{1+\Phi\left(\left\|x_{n+1}-u_{n_{i}+1}\right\|\right)+\left\|x_{n+1}-u_{n+1}\right\|^{2}}\right\}
$$


such that

$$
\lim _{i \rightarrow \infty} \frac{\Phi\left(\left\|x_{n_{i}+1}-u_{n_{i}+1}\right\|\right)}{1+\Phi\left(\left\|x_{n_{i}+1}-u_{n_{i}+1}\right\|\right)+\left\|x_{n_{i}+1}-u_{n_{i}+1}\right\|^{2}}=0 .
$$

Since $0 \leq\left\|x_{n}-u_{n}\right\| \leq M$, it follows that

$$
0 \leq \frac{\Phi\left(\left\|x_{n_{i}+1}-u_{n_{i}+1}\right\|\right)}{1+\Phi(M)+M^{2}} \leq \frac{\Phi\left(\left\|x_{n_{i}+1}-u_{n_{i}+1}\right\|\right)}{1+\Phi\left(\left\|x_{n_{i}+1}-u_{n_{i}+1}\right\|\right)+\left\|x_{n_{i}+1}-u_{n_{i}+1}\right\|^{2}} .
$$

Thus, $\lim _{i \rightarrow \infty} \Phi\left(\left\|x_{n_{i}+1}-u_{n_{i}+1}\right\|\right)=0$. By the strictly increasing continuous function $\Phi$, we obtain that $\lim _{i \rightarrow \infty}\left\|x_{n_{i}+1}-u_{n_{i}+1}\right\|=0$ and so $\lim _{n \rightarrow \infty}\left\|x_{n}-u_{n}\right\|=0$. Using the inequality $\left\|x_{n}-q\right\| \leq\left\|x_{n}-u_{n}\right\|+\left\|u_{n}-q\right\| \rightarrow 0$ as $n \rightarrow \infty$, we know that $x_{n} \rightarrow q$ as $n \rightarrow \infty$. This completes the proof.

Theorem 2.3 Let E be a real Banach space and D be a nonempty closed convex subset of $E$. Let $T: D \rightarrow D$ be a uniformly L-Lipschitz asymptotically pseudocontractive mapping with a sequence $\left\{k_{n}\right\} \subset[1,+\infty)$ such that $\lim _{n \rightarrow \infty} k_{n}=1$. Suppose that $\left\{a_{n}\right\}$ and $\left\{b_{n}\right\}$ are two real sequences in $[0,1]$ satisfying the following conditions:

(a) $a_{n} \rightarrow 0$ as $n \rightarrow \infty$ and $\sum_{n=1}^{\infty} a_{n}=\infty$;

(b) $\sum_{n=1}^{\infty} a_{n}^{2}<\infty$ and $\sum_{n=1}^{\infty} a_{n}\left(k_{n}-1\right)<\infty$;

(c) $\sum_{n=1}^{\infty} a_{n} b_{n}<\infty$.

For any $x_{1} \in D$, let $\left\{x_{n}\right\}$ be the modified Ishikawa iteration defined in (1.6). If $F(T) \neq \emptyset$, $q \in F(T)$ and there exists a strictly increasing continuous function $\Phi:[0,+\infty) \rightarrow[0,+\infty)$ with $\Phi(0)=0$ such that

$$
\left\langle T^{n} x_{n+1}-q, j\left(x_{n+1}-q\right)\right\rangle \leq k_{n}\left\|x_{n+1}-q\right\|^{2}-\frac{\Phi\left(\left\|x_{n+1}-q\right\|\right)}{1+\Phi\left(\left\|x_{n+1}-q\right\|\right)+\left\|x_{n+1}-q\right\|^{2}}, \quad \forall n \geq 1,
$$

where $j\left(x_{n+1}-q\right) \in J\left(x_{n+1}-q\right)$. Then $\left\{x_{n}\right\}$ converges strongly to the fixed point $q$ of $T$.

Proof By Theorem 2.1 and Theorem 2.2, we obtain the proof of Theorem 2.3.

Remark 2.4 Since the condition $\left\langle T^{n} x-q, j(x-q)\right\rangle \leq k_{n}\|x-q\|^{2}-\frac{\Phi(\|x-q\|)}{1+\Phi(\|x-q\|)+\|x-q\|^{2}}$ is weaker than $\left\langle T^{n} x-q, j(x-q)\right\rangle \leq k_{n}\|x-q\|^{2}-\Phi(\|x-q\|)$, Theorem 2.1 and Theorem 2.3 generalize the corresponding results of Zeng [14]. Further, our proof methods are different from those of Zeng [14].

For the sake of convenience, we give the following definitions.

Definition 2.5 A mapping $T: D \rightarrow E$ is said to be weak generalized asymptotically $\varphi$-hemi-contractive with a sequence $\left\{k_{n}\right\} \subset[1,+\infty)$ such that $\lim _{n \rightarrow \infty} k_{n}=1$ if there exists a strictly increasing continuous function $\varphi:[0,+\infty) \rightarrow[0,+\infty)$ with $\varphi(0)=0$ such that, for any $x \in D$ and $y \in F(T)$, there exists $j(x-y) \in J(x-y)$ such that

$$
\left\langle T^{n} x-T^{n} y, j(x-y)\right\rangle \leq k_{n}\|x-y\|^{2}-\frac{\varphi(\|x-y\|)}{1+\varphi(\|x-y\|)+\|x-y\|^{2}}, \quad \forall n \geq 1 .
$$


If the condition (2.17) is replaced by the following inequality:

$$
\left\langle T^{n} x-T^{n} y, j(x-y)\right\rangle \leq k_{n}\|x-y\|^{2}-\varphi(\|x-y\|), \quad \forall n \geq 1,
$$

then $T$ is called a generalized asymptotically $\varphi$-hemi-contractive mapping. Clearly, if $T$ is a generalized asymptotically asymptotically $\varphi$-hemi-contractive, then $T$ must be a weak generalized asymptotically asymptotically $\varphi$-hemi-contractive mapping. However, the converse is not true in general. This can be seen from the following examples.

Example 2.6 Let $E=R$ be the set of real numbers with the usual norm $|\cdot|$ and $D=[0,+\infty)$. Define a mapping $T: D \rightarrow D$ by

$$
T x=\frac{2 x^{3}}{1+2 x^{2}}, \quad \forall x \in D
$$

Then $T$ is a monotonically increasing function with a fixed point $q=0 \in D$. Define two functions $\Phi, \varphi:[0,+\infty) \rightarrow[0,+\infty)$ by $\Phi(t)=\frac{t^{2}}{1+2 t^{2}}$ and $\varphi(t)=t^{2}$, respectively. Then $\Phi$ and $\varphi$ are two strictly increasing continuous functions with $\Phi(0)=\varphi(0)=0$. For all $x \in D$ and $q \in F(T)$, let $k_{n}=1$. Then we obtain that

$$
\begin{aligned}
\left|T^{n} x-T^{n} q\right| \leq|T x| & \leq|x-q|=k_{n}|x-q| \\
\left\langle T^{n} x-T^{n} q, j(x-q)\right\rangle & =\left\langle T^{n} x, j(x-0)\right\rangle \leq\langle T x, j(x-0)\rangle \\
& =\left\langle\frac{2 x^{3}}{1+2 x^{2}}, x\right\rangle=\frac{2 x^{4}}{1+2 x^{2}} \\
& =|x-q|^{2}-\frac{|x-q|^{2}}{1+2|x-q|^{2}} \\
& =k_{n}|x-q|^{2}-\Phi(|x-q|) \\
& =k_{n}|x-q|^{2}-\frac{\varphi(|x-q|)}{1+\varphi(|x-q|)+|x-q|^{2}} .
\end{aligned}
$$

Then $T$ is a generalized asymptotically $\Phi$-hemi-contraction and a weak generalized asymptotically $\varphi$-hemi-contraction.

Example 2.7 Let $E=R$ be the set of real numbers with the usual norm and $R^{+}=[0,+\infty)$. Define a mapping $T: R^{+} \rightarrow R$ by

$$
T x=\frac{x+x^{3}+x^{5 / 2}-x^{1 / 2}}{1+x^{3 / 2}+x^{2}}, \quad \forall x \in R^{+}
$$

Then $T$ has a fixed point $q=0 \in R^{+}$. Define a function $\varphi:[0,+\infty) \rightarrow[0,+\infty)$ by $\varphi(t)=$ $t^{3 / 2}$. Then $\varphi$ is a strictly increasing continuous function with $\varphi(0)=0$. For all $x \in R^{+}$and $q \in F(T)$, let $n=1$ and $k_{n}=1$. Then we have

$$
\begin{aligned}
\langle T x-T q, j(x-q)\rangle & =\left\langle\frac{x+x^{3}+x^{5 / 2}-x^{1 / 2}}{1+x^{3 / 2}+x^{2}}-0, j(x-0)\right\rangle \\
& =\left\langle\frac{x+x^{3}+x^{5 / 2}-x^{1 / 2}}{1+x^{3 / 2}+x^{2}}, x\right\rangle
\end{aligned}
$$




$$
\begin{aligned}
& =\frac{x^{2}+x^{4}+x^{7 / 2}-x^{3 / 2}}{1+x^{3 / 2}+x^{2}} \\
& =x^{2}-\frac{x^{3 / 2}}{1+x^{3 / 2}+x^{2}} \\
& =|x-q|^{2}-\frac{|x-q|^{3 / 2}}{1+|x-q|^{3 / 2}+|x-q|^{2}} \\
& =|x-q|^{2}-\frac{\varphi(|x-q|)}{1+\varphi(|x-q|)+|x-q|^{2}} .
\end{aligned}
$$

Then $T$ is a weak generalized asymptotically $\varphi$-hemi-contraction, but not a generalized asymptotically $\Phi$-hemi-contraction with $n=1$.

\section{Competing interests}

The authors declare that they have no competing interests.

\section{Authors' contributions}

All authors contributed equally in writing this paper and read and approved the final manuscript.

\section{Acknowledgements}

The authors are grateful to Professor Yeol-Je Cho for valuable suggestions which helped to improve the manuscript. This work was supported by Hebei Provincial Natural Science Foundation (Grant No. A2011210033).

Received: 19 August 2012 Accepted: 8 January 2013 Published: 19 April 2013

\section{References}

1. Chang, SS: Iterative approximation problem of fixed point for asymptotically nonexpansive mappings in Banach spaces. Acta Math. Appl. Sin. 24, 236-241 (2001) (Chinese series)

2. Chang, SS, Cho, YJ, Kim, JK: Some results for uniformly L-Lipschitzian mappings in Banach spaces. Appl. Math. Lett. 22, 121-125 (2009)

3. Tang, YC, Liu, LW: Note on some results for asymptotically pseudocontractive mappings and asymptotically nonexpansive mappings. Fixed Point Theory Appl. 2006, Article ID 24978 (2006)

4. Rhoades, BE, Soltuz, SM: The equivalence between the convergences of Ishikawa and Mann iterations for an asymptotically pseudocontractive map. J. Math. Anal. Appl. 283, 681-688 (2003)

5. Cho, YJ, Kang, Jl, Zhou, HY: Approximating common fixed points of asymptotically nonexpansive mappings. Bull. Korean Math. Soc. 42, 661-670 (2005)

6. Chang, SS: Some results for asymptotically pseudocontractive mappings and asymptotically nonexpansive mappings. Proc. Am. Math. Soc. 129, 845-853 (2001)

7. Cho, YJ, Kim, JK, Lan, HY: Three step procedure with errors for generalized asymptotically quasi-nonexpansive mappings in Banach spaces. Taiwan. J. Math. 12, 2155-2178 (2008)

8. Guo, W, Cho, YJ: On the strong convergence of the implicit iterative processes with errors for a finite family of asymptotically nonexpansive mappings. Appl. Math. Lett. 21, 1046-1052 (2008)

9. Zhou, HY, Cho, YJ, Kang, SM: A new iterative algorithm for approximating common fixed points for asymptotically nonexpansive mappings. Fixed Point Theory Appl. 2007, Article ID 64974 (2007)

10. Chang, SS, Cho, YJ, Tian, YX: Strong convergence theorems of Reich type iterative sequence for non-self asymptotically nonexpansive mappings. Taiwan. J. Math. 11, 729-743 (2007)

11. Yao, Y, Cho, YJ: A strong convergence of a modified Krasnoselskii-Mann method for non-expansive mappings in Hilbert spaces. Math. Model. Anal. 15, 265-274 (2010)

12. Osilike, MO: Stability of the Mann and Ishikawa iteration procedures for $\varphi$-strong pseudocontractions and nonlinear equations of the $\varphi$-strongly accretive type. J. Math. Anal. Appl. 227, 319-334 (1998)

13. Chidume, CE, Mutangadura, SA: An example on the Mann iteration method for Lipschitz pseudocontractions. Proc. Am. Math. Soc. 129, 2359-2363 (2001)

14. Zeng, LC: Modified Ishikawa iteration process with errors in Banach spaces. Acta Math. Sin. 47, 219-228 (2004) (Chinese series)

doi:10.1186/1029-242X-2013-188

Cite this article as: Xue and Lv: The convergence of the modified Mann and Ishikawa iterations in Banach spaces. Journal of Inequalities and Applications 2013 2013:188. 